\title{
Rational Construction of Organic Electronic Devices Based on S-Indacene Fragments
}

Hemanth Haridas

Submitted date: 23/03/2021 - Posted date: 24/03/2021

Licence: CC BY-NC-ND 4.0

Citation information: Haridas, Hemanth (2021): Rational Construction of Organic Electronic Devices Based on S-Indacene Fragments. ChemRxiv. Preprint. https://doi.org/10.26434/chemrxiv.14267333.v1

Herein, we report the computational investigation of s-indacene as a viable candidate for the construction of organic electronic devices.

We also investigate the effect of molecular topology on the frontier energy levels of the s-indacene fragments and the possibility of

tuning the frontier energy levels by a rational choice of substituents and bridging groups. The rationale behind the choice of s-indacene

fragments as the basis for the construction of 2D organic electronic devices with tailor-made properties can be extended towards the construction of other 2D covalent organic frameworks with applications in organic electronics and spintronics.

File list (2)

Manuscript_ArXiv.pdf (2.30 MiB) view on ChemRxiv • download file 


\title{
Rational Construction of Organic Electronic Devices based on $s$-indacene fragments
}

\author{
Hemanth $\mathrm{H}^{*}$ \\ * Discipline of Chemistry, Indian Institute of Technology Gandhinagar, Palaj, Gujarat, \\ India - 382355 \\ Email: hemanth.h@iitgn.ac.in
}

\begin{abstract}
Organic electronic devices are sought after for their mechanical and electrical properties and economic viability. Recent advances in the literature point to a plethora of molecules synthesized for applications in organic electronics and solar cells. However, a complete understanding of the design principles for constructing such devices with tailor-made properties is absent in the literature. Herein, we report the computational investigation of s-indacene as a viable candidate for the construction of organic electronic devices. We also investigate the effect of molecular topology on the frontier energy levels of the s-indacene fragments and the possibility of tuning the frontier energy levels by a rational choice of substituents and bridging groups. The rationale behind the choice of s-indacene fragments as the basis for the construction of $2 \mathrm{D}$ organic electronic devices with tailor-made properties can be extended towards the construction of other 2D covalent organic frameworks with applications in organic electronics and spintronics.
\end{abstract}




\section{Introduction}

Graphene, a planar 2D allotrope of carbon with the hexagonal unit cell, have attracted significant attention from the scientific community for its excellent electrical and thermal properties and high tensile strength ${ }^{[1] 4]}$. However, the atomistically thin nature of graphene lattice, and the lack of a bandgap at the Fermi level, leads to a significant leakage current due to quantum effects ${ }^{[5]}$. This issue led to the investigation of other 2D organic electronic devices with suitable electronic properties. Organic electronic devices offer a cleaner and economically viable alternative to the presently used $\mathrm{Si}$ / Ga-As bases semiconducting devices.

Molecules with a significant $\pi$-cloud and aromatic/anti-aromatic nature can form the basis for constructing organic electronic devices with suitable electronic properties ${ }^{[6]}$. Graphene, a 2D material constructed from a hexagonal arrangement of carbon atoms, is an example of an organic electronic device with a significant aromatic character ${ }^{[12]}$. The availability of an unhybridized p orbital per carbon atom provides unmatched mobility for the electrons in the system, owing to the significant delocalization present in the system. However, it is surprising that graphene's unit cell is anti-aromatic in a strict chemical sense due to the availability of two unpaired electrons in the unit cell's two valence bands. Thus, it is intuitive to investigate the rationality behind the construction of 2D materials from various aromatic/anti-aromatic molecules.

$s$-indacene and it's derivatives presents itself as a suitable precursor to developing organic electronic devices due to its unique electronic and structural features [7]9]. The s-indacene core has a mixed aromatic/anti-aromatic character, with the central benzene ring possessing a clar sextet and the two cyclopentadienyl rings having a bi-radical 
character. Computational investigations by various groups reported an assortment of geometric parameters and electronic structures, with few results pointing to geometry with $\mathrm{C}_{2 \mathrm{~h}}$ symmetry and bond alternation ${ }^{[10]}$. In contrast, few others report a geometry with $\mathrm{D}_{2 \mathrm{~h}}$ symmetry and bond delocalization ${ }^{[10+12]}$. The structure with $\mathrm{D}_{2 \mathrm{~h}}$ symmetry stands closer to the core of 1,3,5,7-Tetra-tert-butyl-s-indacene synthesized by Hafner and coworkers in $1986^{[13]}$. It must be noted that the parent $s$-indacene is highly reactive, and undergoes rapid auto-oxidation, and undergoes hydrogenation under mild conditions to form $s$-hydrindacene ${ }^{[14]}$. There are disagreements in the electronic structure of the s-indacene core, with the $\mathrm{C}_{2 \mathrm{~h}}$ geometry preferring a neutral singlet character, while the molecule in $\mathrm{D}_{2 \mathrm{~h}}$ geometry was adopting a neutral triplet character.

The rest of this paper is divided into two parts; In part I of the Results and Discussions, we report the results from electronic structure calculations on the parent $s$ indacene and its derivatives and discuss the effect of substituents and molecular topology on the geometry and frontier energy levels of the molecules. In part II of Results and Discussions, we report the electronic structure calculations on the extended lattices constructed from relevant unit cells and discuss the mapping of the properties of the extended lattice to its constituent molecular fragments.

\section{Computational Methodology}

We performed geometry optimizations for the molecular structures using the Gaussian 09 ${ }^{[15]}$ suite of programs, using a Becke-3 parameter Lee Yand Parr (B-3LYP) hybrid functional and 6-31 $g(\mathrm{~d}, \mathrm{p})$ level of theory for isolated molecules. This combination of theory and basis-set ensures that the calculations are computationally accessible, without the loss of accuracy. We performed a vibrational analysis on the optimized structures to 
ensure that the optimized structures were at a minimum on the potential energy surface (PES). We further evaluated the wavefunctions' stability to ensure the absence of an RHF to UHF instability. The wavefunctions and geometries were reoptimized to the minima when such instabilities were encountered. Such checks are essential for $s$-indacene and its derivatives; since the electronic structure of the parent $s$-indacene is still debated in the scientific community. Density of States (DOS) and Localized Orbital Locator- $\pi\left(\right.$ LOL- $\pi$ ) calculations were performed using Multiwfn ${ }^{[16]}$ package.

Unit cells for Material calculations were generated using the PBC module in Gauss view and exported to Avogadro as a Protein Data Bank (PDB) file. Unit cells were symmetrized and reduced to primitive unit cells using a tolerance of 0.5 in Avogadro. We used the Quantum Espresso ${ }^{[1718]}$ (QE) suite of packages to perform DFT calculations on periodic systems. We employed Local Density Approximation (LDA) pseudopotential (PP) files with PBE xc functional obtained from Quantum Espresso for the periodic cell calculations. All calculations employed a cut-off of 600 Rydberg (Ry) for density and 60 Ry for wavefunctions to ensure smooth convergence in the SCF cycles. The calculations were deemed to have achieved convergence once the errors in forces and energies reached below 1.0d-4 and 1.0d-6, respectively. We also employed a cut-off of $1.0 \mathrm{~d}-12$ for SCF to ensure the convergence of geometry and forces during the optimization cycle. Geometry optimizations were performed on a uniform k-point grid of $12 * 12 * 1$ points.

Electronic structure calculations on the periodic lattice were performed on a k-path defined from $\Gamma-Y-S-\Gamma$ in the first Brillouin zone. 


\section{Results and Discussion}

\section{Electronic structure calculations on isolated molecules}

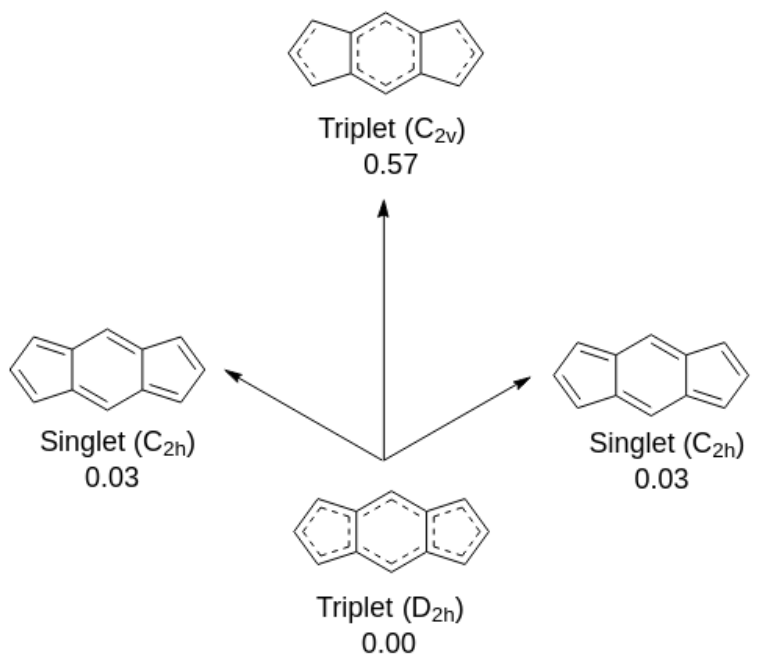

Figure 1: Various transition pathways for parent $s$-indacene. Relative energies of the structures in units of $\mathrm{eV}$ are reported along with the point-group symmetries.

The parent $s$-indacene was constructed using a z-matrix in Gauss View and was optimized to a minimum on the potential energy hypersurface. A vibrational analysis on the optimized structure showed that the neutral singlet structure was a first-order saddle point (354.52i) on the potential energy hypersurface. Upon following the highest imaginary frequency, the optimized structure transitioned from a $D_{2 h}$ symmetry to $\mathrm{C}_{2 \mathrm{~h}}$ symmetry. This transition also converted the initial fully delocalized structure to a fully localized structure, with clear double and single bonds between carbon atoms. However, various experimental and computational studies suggest that substituted $s$ indacenes have a fully delocalized structure with mixed aromatic and anti-aromatic character. To address this, we re-optimized the geometry of the $s$-indacene with a neutral triplet wavefunction, and the vibrational analysis showed that the molecule is at a 


\begin{tabular}{ccccc} 
Point group & Electronic state & Energy & Imaginary modes & Stability \\
\hline $\mathrm{D}_{2 \mathrm{~h}}$ & Singlet & -461.89 & $354.52(1)$ & Unstable \\
$\mathrm{C}_{2 \mathrm{~h}}$ & Singlet & -461.88 & $-(0)$ & Stable \\
$\mathrm{C}_{2 \mathrm{v}}$ & Triplet & -461.86 & $-(0)$ & Stable
\end{tabular}

Table 1: Various descriptors for the s-indacene geometries considered in the study. All energies are presented in units of Hatree.

minimum on the potential energy surface, with a $C_{2 v}$ symmetry. We further confirmed the wavefunction's stability, and the wavefunction was found to be stable under perturbations. Our calculations suggest that the parent $s$-indacene is a neutral biradical at its electronic ground state, with a significant bond-delocalization within the structure. This observation corroborates with the previous studies by Nendel and coworkers ${ }^{[10]}$, where they studied the aromaticity of $s$-indacene using ab-initio methods. It must be noted that Nendel and coworkers investigated the potential energy landscape for a singlet ground state, while our results are for triplet ground state. We present the final energies, number of imaginary frequencies and stability of the wavefunctions in Table 1. We observe that the neutral singlet forms of $s$-indacene with $\mathrm{D}_{2 \mathrm{~h}}$ and $\mathrm{C}_{2 \mathrm{~h}}$ symmetries are comparable in energy. However, the $\mathrm{D}_{2 \mathrm{~h}}$ form with closed-shell singlet character is a transition state connecting the $\mathrm{C}_{2 \mathrm{~h}}$ minima of the potential energy surface. We note that the neutral triplet form of s-indacene is a minimum on the potential energy surface, with vibrational analysis confirming the structural stability of the optimized geometry. Thus, we can conclude that structure with $\mathrm{C}_{2 \mathrm{~h}}$ symmetry is the electronic minima of the $s$-indacene moiety, while $\mathrm{C}_{2 \mathrm{v}}$ symmetry with biradical (triplet) character is a local minimum on the potential energy hypersurface at the height of $13.16 \mathrm{kcal} / \mathrm{mol}$. 


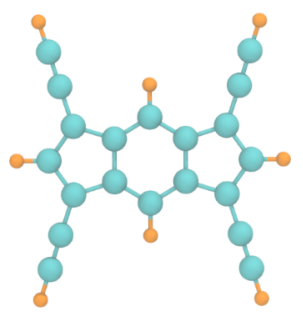

TASI-1

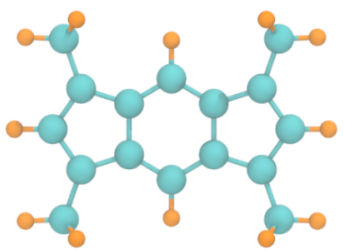

TMSI-1

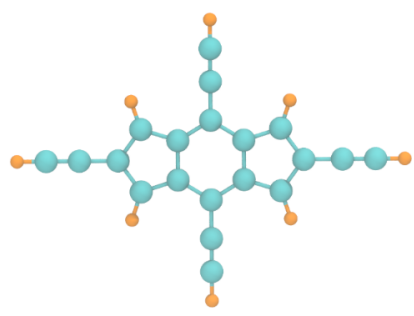

TASI-2

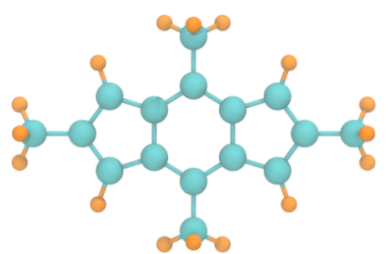

TMSI-2

Figure 2: Representative structures for TASI-1,TASI-2, TMSI-1 and TMSI-2. Carbon atoms are coloured green, and Hydrogen atoms are coloured orange. All figures are prepared using VMD

Having concluded the electronic nature of s-indacene, we investigated the effect of perturbations on the electronic structure of the s-indacene core by symmetrically introducing four electron-donating and four electron-withdrawing substituents. We introduced four methyl groups onto the s-indacene core to construct tetra-methyl substituted s-indacenes TMSI-1 and TMSI-2, and four acetylene groups to construct tetra-acetylene substituted s-indacenes TASI-1 and TASI-2. Representative structures for TASI-1, TASI-2, TMSI-1 and TMSI-2 are presented in Figure 2. We performed geometry optimizations and vibrational analyses on TASI-1, TASI-2, TMSI-1, and TMSI-2. We observed that TMSI-1, TMSI-2, TASI-1, and TASI-2 retained significant delocalization of electrons in the system. This observation presented an interesting conundrum, where we can selectively stabilize the electronic state of a molecule to suit our needs via the introduction 
(a)

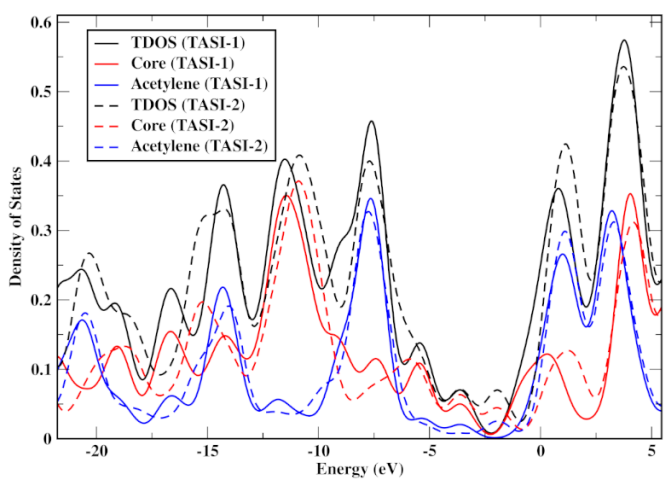

(b)

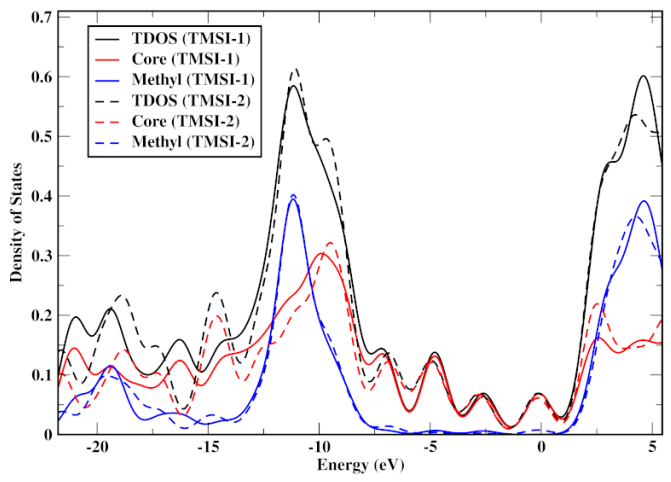

Figure 3: Density of States (DOS) plots for (a) TASI-1,2 and (b) TMSI-1,2. All energies are presented in units of eV.

of suitable substituents on the structural core. We also note that differences in frontier energy levels and stable minima in the potential energy surface are correlated with the location of substitution in the $s$-indacene core. It was observed that introducting substituents along the $\mathrm{C}_{2}$ and $\mathrm{C}_{2}$ axes of the $s$-indacene core results in a reduction in the symmetry of the optimized geometry. Introduction of substituents along the symmetry axes broke the initial $\mathrm{D}_{2 \mathrm{~h}}$ symmetry for TASI-2 and TMSI-2, and transitioned to $\mathrm{C}_{2 \mathrm{~h}}$ symmetry during geometry optimizations. However, no such effects were observed for TMSI-1 and TASI-1, where the substituents were introduced away from the symmetry axes. We present DOS plots for TASI and TMSI molecules in Figure 3. The frontier energy levels of TMSI and TASI molecules are dominated by contributions from the $s$ indacene core. In TASI molecules, very small contributions from acetylene groups are present in the frontier molecular orbitals. In contrast, the contributions from methyl groups to the frontier orbitals of TMSI molecules are negligible, as seen in Figure 3.

We constructed two tetramers from four TASI- 2 molecules, where TASI-E1 is constructed by symmetric acetylene bridges at positions $2,4,6$ and 8 positions, and TASI-E2 


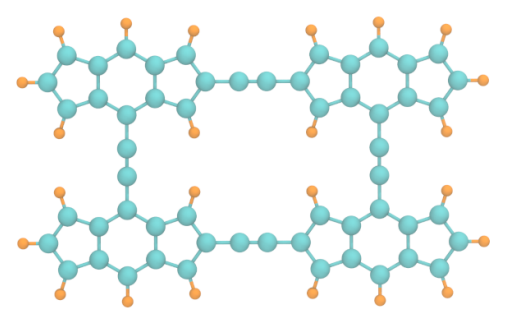

TASI-E1

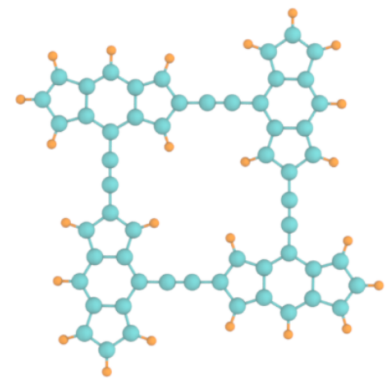

TASI-E2

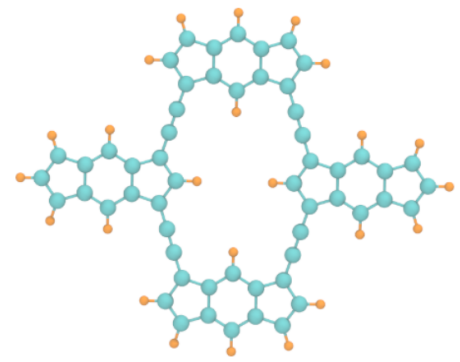

TASI-E3

Figure 4: Representative structures for TASI-E1,TASI-E2 and TASI-E3. Carbon atoms are coloured green, and Hydrogen atoms are coloured orange. All figures are prepared using VMD [19]

is constructed by asymmetric acetylene bridges at the same positions respectively. TASIE3 was constructed from four TASI-1 molecules by acetylene bridges on 1,3,5 and 7 positions on the s-indacene core. We optimized the geometries for the tetramers constructed, and vibrational analyses were performed to confirm the stability of the optimized geometries. We observed that TASI-E1 transitioned from $D_{2 h}$ symmetry to $C_{2 v}$ symmetry during the geometry optimization, while TASI-E2 and TASI-E3 were found to confirm to $\mathrm{C}_{4 \mathrm{~h}}$ and $\mathrm{D}_{2 \mathrm{~h}}$ symmetries respectively. Representative structures for TASI-E1, TASI-E2 and TASI-E3 are presented in Figure 4.

We observe that contributions from $s$-indacene core dominates the Fermi levels for TASI-E1, TASI-E2 and TASI-E3. Contributions from four acetylene bridges were found to be $\approx 11.7 \%$, with four $s$-indacene cores contributing $\approx 89.3 \%$ towards the Fermi level.

Harmonic Oscillator Model of Aromaticity (HOMA) analysis is a geometric aromaticity index routinely used to classify molecules as aromatic, ant-aromatic or non-aromatic, with benzene as the unit of comparison [20|21]. HOMA value of 1 corresponds to perfect aromaticty, while negative values denote strong anti-aromatic nature, and values near 


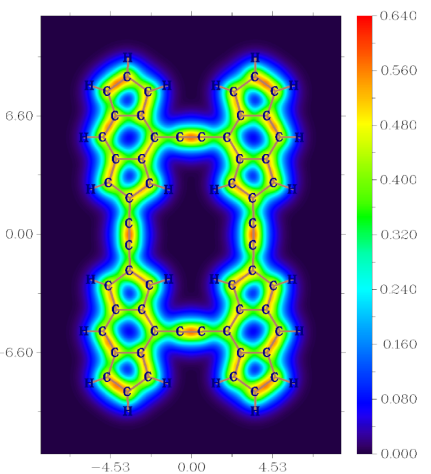

TASI-E1

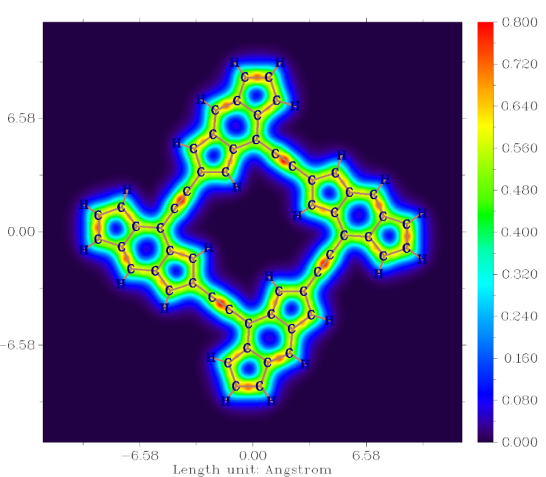

TASI-E2

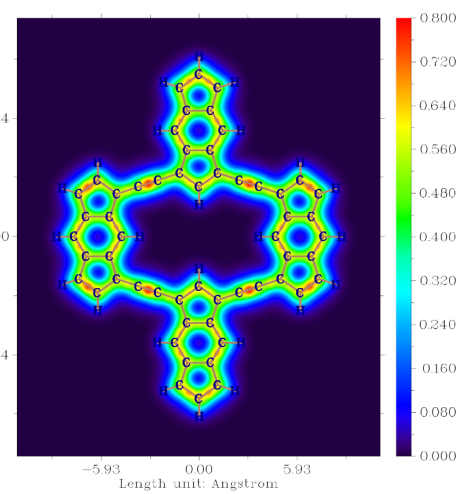

TASI-E3

Figure 5: Path of electron delocalization in TASI-E1,TASI-E2 and TASI-E3 obtained from LOL- $\pi$ analysis. The preferred delocalization path is coloured as orange-red. All figures are prepared using Multiwfn [16]

to 0 correspond to non-aromoatic nature of the molecule. HOMA values for the central rings in TASI-E1, TASI-E2 and TASI-E3 are $-0.33,-0.26$ and -0.25 respectively. We conclude from the HOMA values that central rings in TASI-E1, TASI-E2 and TASI-E3 are weakly anti-aromatic. The central rings in TASI-E1, TASI-E2 and TASI-E3 contains 24 $\pi$ electrons, and would be classified as anti-aromatic acording to Hückel theory. However, from LOL- $\pi$ analysis of the structures, we observed that while TASI-E1 behaves as four isolated TASI-1 molecules with minimal delocalization, TASI-E2 and TASI-E3 were found to have significant electron-delocalization, as observed from Figure 5. We note that while TASI-E1 and TASI-E2 are constructed from identical units, the difference in the topology of the structures resulted in vastly different chemical and electronic properties. It must also be noted that TASI-E2 and TASI-E3, constructed from structurally different units have nearly identical HOMA values and electron-delocalization. We present the point group, FMOs, Band-gap and HOMA values for the structures in Table 2. It is observed that band-gap for TASI-E1 fall within the range of Near-IR(A) 


\begin{tabular}{cccccc} 
Molecule ID & Point group & HOMO & LUMO & Band-gap & HOMA \\
\hline TASI-E1 & $\mathrm{D}_{2 \mathrm{~h}}$ & -4.69 & -3.64 & 1.05 & -0.33 \\
TASI-E2 & $\mathrm{C}_{4 \mathrm{~h}}$ & -5.09 & -3.31 & 1.78 & -0.26 \\
TASI-E3 & $\mathrm{D}_{2 \mathrm{~h}}$ & -4.71 & -4.02 & 0.69 & -0.25 \\
\hline
\end{tabular}

Table 2: Various descriptors for tetramers constructed from TASI-1 and TASI-2 units respectively. HOMO and LUMO levels are reported in units of eV. Band-gap is presented in units of eV.

region of the spectrum at $1.05 \mathrm{eV}(1180.8 \mathrm{~nm})$, while TASI-E2 is predicted to have an adsorption peak centered around $1.78 \mathrm{eV}(692.6 \mathrm{~nm})$ in the visible region, and TASIE3 is expected to have an adsorption peak centered around $0.69 \mathrm{eV}(1796.8 \mathrm{~nm})$, in Near-IR(B) region of the electromagnetic spectrum.

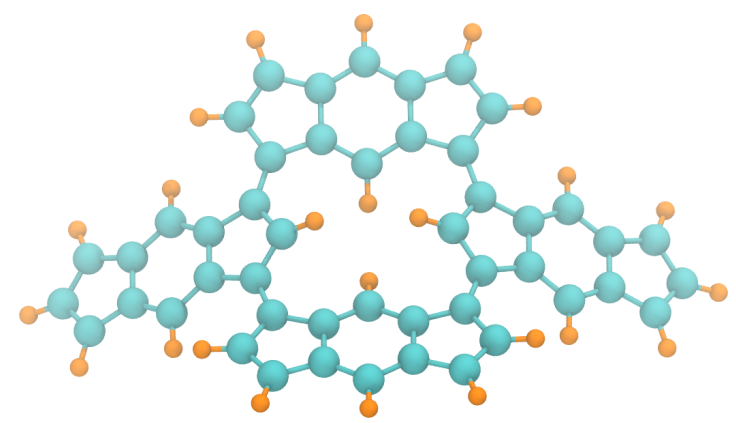

Figure 6: Optimized structure for TMSI-E1. Carbon atoms are coloured green, and Hydrogen atoms are coloured orange. Figures prepared using VMD [19]

TMSI-E1 tetramer was constructed by connecting four TMSI-2 units via C-C bridges at 1,3,5 and 7 positions. We restrict the analyses of the tetramers generated by TMSI-2, since the tetramer generated by TMSI-1 would suffer from steric crowding and would not generate a stable 2D tiling of the surface. Geometry optimization and vibrational analysis of TMSI-1 tetramer showed that the optimized structure was a first-order sad- 
dle point on the potential energy surface. Reoptimizing the structure by chasing the highest imaginary mode resulted in the doming of the tetrameric structure. Doming of TMSI-E1 was accompanied by a lowering in the symmetry from $\mathrm{D}_{2 \mathrm{~h}}$ to $\mathrm{C}_{2 \mathrm{v}}$. Doming of the cyclic structures have previously been reported for porphyrins and related porphyrinoids $[22-24]$, where two close-lying energy states with the same symmetry (JahnTeller ${ }^{[25]}$ ) or different symmetries (pseudo-Jahn-Teller ${ }^{[26]}$ ) undergo mixing to distort the molecular geometry, thereby increasing the energy gap between the HOMO and LUMO orbitals. Tracing the origin of the geometric distortion in TMSI-E1 shows that HOMO, HOMO-1 and LUMO orbitals with symmetries $\mathrm{A}_{\mathrm{u}}, \mathrm{B}_{1 \mathrm{~g}}$ and $\mathrm{B}_{2 \mathrm{~g}}$ underwent mixing to split the levels as $A_{2}, B_{2}$ and $B_{2}$ respectively. Optimized structure for TMSI-E1 is presented in Figure 6.

We study the extended network (TASI-EL) generated by periodic tiling of the TASI-E3 molecule in the subsequent section to understand the electronic and structural features of the $2 \mathrm{D}$ sheet generated by the tiling process. Electronic structure calculations on extended systems are restricted to TASI-E3 unit cell, due the high symmetry of TASI-E3. We also map the bands in the TASI periodic structure to the molecular orbitals in the TASI-E3 unit cell to better understand the connections between the electronic structure of the periodic lattice and the corresponding molecular fragment.

\section{Electronic structure calculations on extended structures}

The geometry of TASI-EL was optimized as described in the methodology section. The optimized cell belonged to the orthorhombic lattice, with a space group of $\mathrm{Cmmm}$, while the primitive cell belonged to the monoclinic lattice. Optimized structure for TASI-EL is presented in Figure 7. Cell parameters for conventional (primitive) cells are a = 


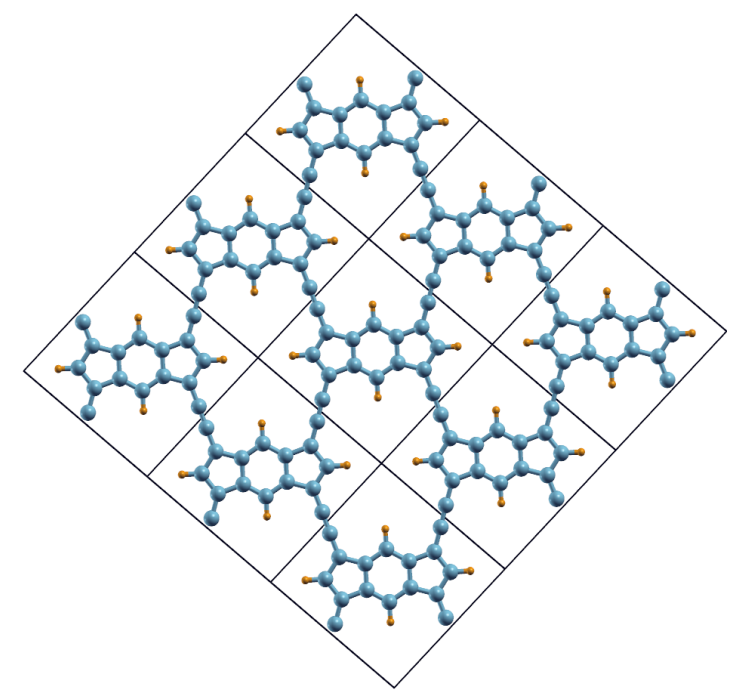

Figure 7: Optimized structure for 2D lattice generated by TASI-E3 (TASI-EL). Carbon atoms are coloured green, and Hydrogen atoms are coloured orange. Unit cell boundaries are marked as black lines. Figures prepared using Xcrysden [27].

$12.22726(8.83540) \AA, b=12.75741(8.83540) \AA, c=29.97583$ (29.97583) $\AA, \alpha=$ $90.0(90.0)^{\circ}, \beta=90.0(90.0)^{\circ}$ and $\gamma=90.0(92.43117)^{\circ}$.

Analysis of the geometric parameters show that root-mean-square (RMS) deviations in bond lengths and bond angles between TASI-E3 and TASI-EL are $0.01 \AA$ and 2.50 degrees. Larger deviation in the bond angles is due to the constrained optimization in the periodic lattice, which reduces the degrees of freedom available for the unit cell. The central pore in TASI-EL and TASI-E3 are identical to an ellipse with minor axis lying along the five-membered rings, and major axis lying along the six-membered rings. This would create a structural anisotropy in the extended lattice, leading to anisotropic electron transport in the system, upon introduction of suitable substituents in the unit cell.

From the band structure calculations on the extended lattice generated by TASI, we observed that TASI-EL has a direct bandgap of $0.73 \mathrm{eV}$, which corresponds to optical 


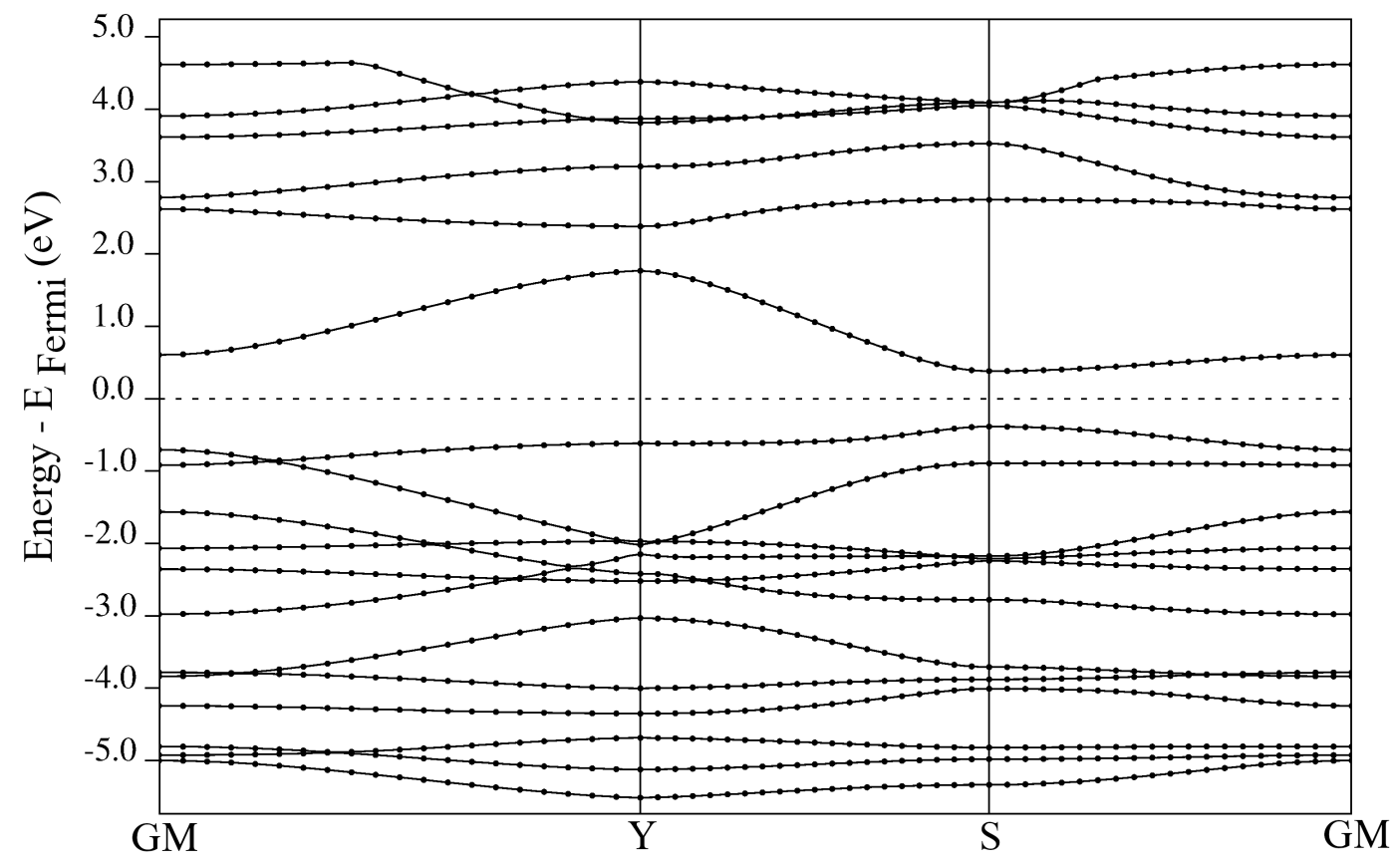

Figure 8: Electronic Band structure for TASI extended structure (TASI-EL). All energy levels are shifted with respect to Fermi level.

adsorption at around $173 \mathrm{THz}$, or in the NIR-B region. This observation corroborates with the electronic band-gap observed for the TASI-E3 tetramer $(0.69 \mathrm{eV})$ discussed in Section 3.1. We present the band structure for TASI-EL in Figure 8.

\section{Conclusions}

In summary, the present study shows that molecules with an $s$-indacene core are suitable building blocks for constructing organic electronic devices. The present study sheds light on the design principles for constructing organic electronic devices with tailor-made properties. We have explored the chemical reasoning behind the choice of $s$-indacene as a precursor for constructing an extended 2D organic sheet and have 
validated the choice by using electronic structure calculations on various molecules and materials constructed from the unit-cell. We also investigated the effect of molecular topology on the electronic structure of organic lattices and showed that such perturbations could effectively tune the electronic properties of the extended structure. We also note a Dirac Cone at $-1.0 \mathrm{eV}$ in the band structure of the TASI lattice. This observation points to the fact that we could, in principle, realize topologically non-trivial materials in 2D lattices constructed from organic building blocks. It would be interesting to study the effect of isolobal substitutions of $\mathrm{CH}$ groups by $\mathrm{N}$ on the electronic structure and geometries of the $s$-indacene derivatives.

\section{Acknowledgements}

H.H thanks Dr. Sairam S. Mallajosyula and Dr. Gopinathan Kalon for constructive discussions and relevant pointers. SRF Fellowship from Ministry of Human Resources Development, Govt. of India, and Infrastructure and Facilities from Indian Institute of Technology Gandhinagar are gratefully acknowledged.

\section{References}

[1] A. K. Geim, Science, 2009, 324, 1530-1534.

[2] A. K. Geim and K. S. Novoselov, Nature Materials, 2007, 6, 183-191.

[3] G. Lalwani, A. M. Henslee, B. Farshid, L. Lin, F. K. Kasper, Y. X. Qin, A. G. Mikos and B. Sitharaman, Biomacromolecules, 2013, 14, 900-909.

[4] D. Akinwande, L. Tao, Q. Yu, X. Lou, P. Peng and D. Kuzum, IEEE Nanotechnology Magazine, 2015, 9, 6-14. 
[5] A. Lanzara, Physics, 2015, 8, 9.

[6] J. J. Dressler, M. Teraoka, G. L. Espejo, R. Kishi, S. Takamuku, C. J. Gomez-Garcia, L. N. Zakharov, M. Nakano, J. Casado and M. M. Haley, Nature Chemistry, 2018, 10, 1134-1140.

[7] R. D. Breukers, A. P. Middleton, S. Janssens, S. G. Raymond, D. J. Clarke, A. J. Kay and G. J. Smith, Optical Processes in Organic Materials and Nanostructures II, 2013, pp. $82-89$.

[8] M. A. Majewski, P. J. Chmielewski, A. Chien, Y. Hong, T. Lis, M. Witwicki, D. Kim, P. M. Zimmerman and M. Stępień, Chem. Sci., 2019, 10, 3413-3420.

[9] T. Jousselin-Oba, P. E. Deal, A. G. Fix, C. K. Frederickson, C. L. Vonnegut, A. Yassar, L. N. Zakharov, M. Frigoli and M. M. Haley, Chemistry - An Asian Journal, 2019, $14,1737-1744$.

[10] M. Nendel, B. Goldfuss, K. Houk and K. Hafner, Journal of Molecular Structure: THEOCHEM, 1999, 461-462, 23-28.

[11] R. H. Hertwig, M. C. Holthausen, W. Koch and Z. B. Maksić, Angewandte Chemie International Edition in English, 1994, 33, 1192-1194.

[12] R. Soriano Jartín, A. Ligabue, A. Soncini and P. Lazzeretti, The Journal of Physical Chemistry A, 2002, 106, 11806-11814.

[13] K. Hafner, B. Stowasser, H.-P. Krimmer, S. Fischer, M. C. Böhm and H. J. Lindner, Angewandte Chemie International Edition in English, 1986, 25, 630-632.

[14] K. Hafner, K. H. Häfner, C. König, M. Kreuder, G. Ploss, G. Schulz, E. Sturm and 
K. H. Vöpel, Angewandte Chemie International Edition in English, 1963, 2, 123134.

[15] M. J. Frisch, G. W. Trucks, H. B. Schlegel, G. E. Scuseria, M. A. Robb, J. R. Cheeseman, G. Scalmani, V. Barone, G. A. Petersson, H. Nakatsuji, X. Li, M. Caricato, A. V. Marenich, J. Bloino, B. G. Janesko, R. Gomperts, B. Mennucci, H. P. Hratchian, J. V. Ortiz, A. F. Izmaylov, J. L. Sonnenberg, D. Williams-Young, F. Ding, F. Lipparini, F. Egidi, J. Goings, B. Peng, A. Petrone, T. Henderson, D. Ranasinghe, V. G. Zakrzewski, J. Gao, N. Rega, G. Zheng, W. Liang, M. Hada, M. Ehara, K. Toyota, R. Fukuda, J. Hasegawa, M. Ishida, T. Nakajima, Y. Honda, O. Kitao, H. Nakai, T. Vreven, K. Throssell, J. A. Montgomery, Jr., J. E. Peralta, F. Ogliaro, M. J. Bearpark, J. J. Heyd, E. N. Brothers, K. N. Kudin, V. N. Staroverov, T. A. Keith, R. Kobayashi, J. Normand, K. Raghavachari, A. P. Rendell, J. C. Burant, S. S. Iyengar, J. Tomasi, M. Cossi, J. M. Millam, M. Klene, C. Adamo, R. Cammi, J. W. Ochterski, R. L. Martin, K. Morokuma, O. Farkas, J. B. Foresman and D. J. Fox, Gaussian 09, Revision A.02, 2016, Gaussian Inc. Wallingford CT.

[16] T. Lu and F. Chen, Journal of Computational Chemistry, 2012, 33, 580-592.

[17] P. Giannozzi, S. Baroni, N. Bonini, M. Calandra, R. Car, C. Cavazzoni, D. Ceresoli, G. L. Chiarotti, M. Cococcioni, I. Dabo, A. Dal Corso, S. de Gironcoli, S. Fabris, G. Fratesi, R. Gebauer, U. Gerstmann, C. Gougoussis, A. Kokalj, M. Lazzeri, L. Martin-Samos, N. Marzari, F. Mauri, R. Mazzarello, S. Paolini, A. Pasquarello, L. Paulatto, C. Sbraccia, S. Scandolo, G. Sclauzero, A. P. Seitsonen, A. Smogunov, P. Umari and R. M. Wentzcovitch, Journal of Physics: Condensed Matter, 2009, 21, 395502 (19pp). 
[18] P. Giannozzi, O. Andreussi, T. Brumme, O. Bunau, M. B. Nardelli, M. Calandra, R. Car, C. Cavazzoni, D. Ceresoli, M. Cococcioni, N. Colonna, I. Carnimeo, A. D. Corso, S. de Gironcoli, P. Delugas, R. A. D. Jr, A. Ferretti, A. Floris, G. Fratesi, G. Fugallo, R. Gebauer, U. Gerstmann, F. Giustino, T. Gorni, J. Jia, M. Kawamura, H.-Y. Ko, A. Kokalj, E. Küçükbenli, M. Lazzeri, M. Marsili, N. Marzari, F. Mauri, N. L. Nguyen, H.-V. Nguyen, A. O. de-la Roza, L. Paulatto, S. Poncé, D. Rocca, R. Sabatini, B. Santra, M. Schlipf, A. P. Seitsonen, A. Smogunov, I. Timrov, T. Thonhauser, P. Umari, N. Vast, X. Wu and S. Baroni, Journal of Physics: Condensed Matter, 2017, 29, 465901.

[19] W. Humphrey, A. Dalke and K. Schulten, Journal of Molecular Graphics, 1996, 14, 33-38.

[20] J. Kruszewski and T. Krygowski, Tetrahedron Letters, 1972, 13, 3839-3842.

[21] T. M. Krygowski, H. Szatylowicz, O. A. Stasyuk, J. Dominikowska and M. Palusiak, Chemical Reviews, 2014, 114, 6383-6422.

[22] M. P. Austeria, P. D. Pancharatna and M. M. Balakrishnarajan, European Journal of Inorganic Chemistry, 2014, 2014, 3200-3207.

[23] Z. Zhou, M. Shen, C. Cao, Q. Liu and Z. Yan, Chemistry-A European Journal, 2012, 18, 7675-7679.

[24] C. Rovira, K. Kunc, J. Hutter, P. Ballone and M. Parrinello, The Journal of Physical Chemistry A, 1997, 101, 8914-8925.

[25] R. Englman and R. Englman, The Jahn-Teller effect in molecules and crystals, WileyInterscience New York, 1972. 
[26] I. B. Bersuker, Chemical Reviews, 2013, 113, 1351-1390.

[27] A. Kokalj, Journal of Molecular Graphics and Modelling, 1999, 17, 176-179. 


\title{
Supporting Information File
}

Rational Construction of Organic Electronic Devices based on $s$-indacene fragments

\author{
Hemanth $\mathrm{H}^{*}$ \\ * Discipline of Chemistry, Indian Institute of Technology Gandhinagar, Palaj, Gujarat, \\ India - 382355
}

Email: hemanth.h@iitgn.ac.in 


\section{Optimized coordinates for TASI-1}

$\begin{array}{lrrr}\text { C } & 0.000000 & 0.716973 & 1.194608 \\ \text { C } & 0.000000 & -0.716973 & 1.194608 \\ \text { C } & 0.000000 & -1.440624 & 0.000000 \\ \text { C } & 0.000000 & -0.716973 & -1.194608 \\ \text { C } & 0.000000 & 0.716973 & -1.194608 \\ \text { C } & 0.000000 & 1.440624 & 0.000000 \\ \text { C } & 0.000000 & 1.147929 & 2.570096 \\ \text { C } & 0.000000 & 0.000000 & 3.389602 \\ \text { C } & 0.000000 & -1.147929 & 2.570096 \\ \text { H } & 0.000000 & -2.527480 & 0.000000 \\ \text { H } & 0.000000 & 2.527480 & 0.000000 \\ \text { H } & 0.000000 & 0.000000 & 4.470474 \\ \text { C } & 0.000000 & 1.147929 & -2.570096 \\ \text { C } & 0.000000 & -1.147929 & -2.570096 \\ \text { C } & 0.000000 & 0.000000 & -3.389602 \\ \text { H } & 0.000000 & 0.000000 & -4.470474 \\ \text { C } & 0.000000 & 2.486831 & 3.010921 \\ \text { C } & 0.000000 & -2.486831 & 3.010921 \\ \text { C } & 0.000000 & 2.486831 & -3.010921 \\ \text { C } & 0.000000 & -2.486831 & -3.010921 \\ \text { C } & 0.000000 & 3.644168 & 3.375215 \\ \text { H } & 0.000000 & 4.657512 & 3.704593 \\ \text { C } & 0.000000 & -3.644168 & 3.375215 \\ \text { H } & 0.000000 & -4.657512 & 3.704593 \\ \text { C } & 0.000000 & 3.644168 & -3.375215 \\ \text { H } & 0.000000 & 4.657512 & -3.704593 \\ \text { C } & 0.000000 & -3.644168 & -3.375215 \\ \text { H } & 0.000000 & -4.657512 & -3.704593\end{array}$

\section{Optimized coordinates for TASI-2}

$\begin{array}{lrrr}\text { C } & -0.038144 & 3.387241 & 0.000000 \\ \text { C } & 1.129452 & 2.528907 & 0.000000 \\ \text { C } & 0.712503 & 1.206137 & 0.000000 \\ \text { C } & -0.740144 & 1.205552 & 0.000000 \\ \text { C } & -1.168233 & 2.577189 & 0.000000 \\ \text { C } & -0.004555 & 4.804240 & 0.000000\end{array}$




$\begin{array}{lrrr}\text { H } & 2.153739 & 2.879472 & 0.000000 \\ \mathrm{H} & -2.195021 & 2.917236 & 0.000000 \\ \mathrm{C} & -1.462631 & 0.018047 & 0.000000 \\ \mathrm{C} & 1.462631 & -0.018047 & 0.000000 \\ \mathrm{C} & -0.712503 & -1.206137 & 0.000000 \\ \mathrm{C} & 0.740144 & -1.205552 & 0.000000 \\ \mathrm{C} & -2.885095 & -0.002049 & 0.000000 \\ \mathrm{C} & 2.885095 & 0.002049 & 0.000000 \\ \mathrm{C} & -1.129452 & -2.528907 & 0.000000 \\ \mathrm{C} & 1.168233 & -2.577189 & 0.000000 \\ \mathrm{C} & 0.038144 & -3.387241 & 0.000000 \\ \mathrm{H} & -2.153739 & -2.879472 & 0.000000 \\ \mathrm{H} & 2.195021 & -2.917236 & 0.000000 \\ \mathrm{C} & 0.004555 & -4.804240 & 0.000000 \\ \mathrm{C} & 0.038144 & 6.014945 & 0.000000 \\ \mathrm{H} & 0.070001 & 7.079622 & 0.000000 \\ \mathrm{C} & -0.038144 & -6.014945 & 0.000000 \\ \mathrm{H} & -0.070001 & -7.079622 & 0.000000 \\ \mathrm{C} & -4.095651 & -0.029735 & 0.000000 \\ \mathrm{C} & 4.095651 & 0.029735 & 0.000000 \\ \mathrm{H} & -5.161481 & -0.048833 & 0.000000 \\ \mathrm{H} & 5.161481 & 0.048833 & 0.000000\end{array}$

\section{Optimized coordinates for TMSI-1}

$\begin{array}{lrrr}\text { C } & 3.373224 & 0.000000 & 0.000000 \\ \text { C } & 2.566366 & 1.153259 & 0.000000 \\ \text { C } & 1.201636 & 0.723781 & 0.000000 \\ \text { C } & 1.201636 & -0.723781 & 0.000000 \\ \text { C } & 2.566366 & -1.153259 & 0.000000 \\ \text { C } & 0.000000 & 1.437313 & 0.000000 \\ \text { C } & 0.000000 & -1.437313 & 0.000000 \\ \text { C } & -1.201636 & 0.723781 & 0.000000 \\ \text { C } & -1.201636 & -0.723781 & 0.000000 \\ \text { C } & -2.566366 & 1.153259 & 0.000000 \\ \text { C } & -2.566366 & -1.153259 & 0.000000 \\ \text { C } & -3.373224 & 0.000000 & 0.000000 \\ \text { C } & 3.052909 & 2.568112 & 0.000000 \\ \text { C } & 3.052909 & -2.568112 & 0.000000\end{array}$




$\begin{array}{lrrr}\text { C } & -3.052909 & 2.568112 & 0.000000 \\ \text { C } & -3.052909 & -2.568112 & 0.000000 \\ \text { H } & 4.457514 & 0.000000 & 0.000000 \\ \text { H } & -4.457514 & 0.000000 & 0.000000 \\ \text { H } & 4.145197 & 2.612405 & 0.000000 \\ \text { H } & 2.694617 & 3.116801 & 0.880095 \\ \text { H } & 2.694617 & 3.116801 & -0.880095 \\ \text { H } & 4.145197 & -2.612405 & 0.000000 \\ \text { H } & 2.694617 & -3.116801 & -0.880095 \\ \text { H } & 2.694617 & -3.116801 & 0.880095 \\ \text { H } & -4.145197 & 2.612405 & 0.000000 \\ \text { H } & -2.694617 & 3.116801 & -0.880095 \\ \text { H } & -2.694617 & 3.116801 & 0.880095 \\ \text { H } & -4.145197 & -2.612405 & 0.000000 \\ \text { H } & -2.694617 & -3.116801 & 0.880095 \\ \text { H } & -2.694617 & -3.116801 & -0.880095 \\ \text { H } & 0.000000 & 2.526158 & 0.000000 \\ \text { H } & 0.000000 & -2.526158 & 0.000000\end{array}$

\section{Optimized coordinates for TMSI-2}

$\begin{array}{lrrr}\text { C } & -0.016674 & 1.389681 & 0.000000 \\ \text { C } & -1.264650 & 0.633791 & 0.000000 \\ \text { C } & -1.271447 & -0.740660 & 0.000000 \\ \text { C } & 0.016674 & -1.389681 & 0.000000 \\ \text { C } & 1.264650 & -0.633791 & 0.000000 \\ \text { C } & 1.271447 & 0.740660 & 0.000000 \\ \text { C } & -0.352017 & 2.730869 & 0.000000 \\ \text { C } & -1.799053 & 2.872654 & 0.000000 \\ \text { C } & -2.342492 & 1.612438 & 0.000000 \\ \text { H } & 0.342336 & 3.564647 & 0.000000 \\ \text { H } & -3.402094 & 1.386740 & 0.000000 \\ \text { C } & 2.342492 & -1.612438 & 0.000000 \\ \text { H } & 3.402094 & -1.386740 & 0.000000 \\ \text { C } & 1.799053 & -2.872654 & 0.000000 \\ \text { C } & 0.352017 & -2.730869 & 0.000000 \\ \text { H } & -0.342336 & -3.564647 & 0.000000 \\ \text { C } & -2.529994 & 4.181748 & 0.000000 \\ \text { H } & -2.278023 & 4.786219 & 0.880554\end{array}$




$\begin{array}{lrrr}\mathrm{H} & -2.278023 & 4.786219 & -0.880554 \\ \mathrm{H} & -3.612980 & 4.029519 & 0.000000 \\ \mathrm{C} & 2.529994 & 1.564019 & 0.000000 \\ \mathrm{H} & 3.426383 & 0.942149 & 0.000000 \\ \mathrm{H} & 2.565873 & 2.216037 & -0.880283 \\ \mathrm{H} & 2.565873 & 2.216037 & 0.880283 \\ \mathrm{C} & -2.529994 & -1.564019 & 0.000000 \\ \mathrm{H} & -2.565873 & -2.216037 & -0.880283 \\ \mathrm{H} & -2.565873 & -2.216037 & 0.880283 \\ \mathrm{H} & -3.426383 & -0.942149 & 0.000000 \\ \mathrm{C} & 2.529994 & -4.181748 & 0.000000 \\ \mathrm{H} & 2.278023 & -4.786219 & -0.880554 \\ \mathrm{H} & 3.612980 & -4.029519 & 0.000000 \\ \mathrm{H} & 2.278023 & -4.786219 & 0.880554\end{array}$

\section{Optimized coordinates for TASI-EL}

Note: Coordinates provided are in Fractional Coordinates

$\begin{array}{lrrr}\text { H } & 0.852014258 & 0.852014258 & -0.000000000 \\ \text { H } & -0.852014258 & -0.852014258 & 0.000000000 \\ \text { H } & -0.706998296 & -0.293001704 & 0.000000000 \\ \text { H } & 0.706998296 & 0.293001704 & -0.000000000 \\ \text { C } & -0.233097481 & -0.233097481 & 0.000000000 \\ \text { C } & 0.233097481 & 0.233097481 & -0.000000000 \\ \text { C } & -0.202847004 & -0.391467032 & 0.000000000 \\ \text { C } & -0.348509313 & -0.464878963 & -0.000000000 \\ \text { C } & -0.062158892 & -0.465196909 & 0.000000000 \\ \text { C } & 0.202847004 & 0.391467032 & -0.000000000 \\ \text { C } & 0.348509313 & 0.464878963 & -0.000000000 \\ \text { C } & 0.062158892 & 0.465196909 & -0.000000000 \\ \text { C } & -0.391467032 & -0.202847004 & 0.000000000 \\ \text { C } & -0.464878963 & -0.348509313 & 0.000000000 \\ \text { C } & -0.465196909 & -0.062158892 & 0.000000000 \\ \text { C } & 0.391467032 & 0.202847004 & 0.000000000 \\ \text { C } & 0.464878963 & 0.348509313 & -0.000000000 \\ \text { C } & 0.465196909 & 0.062158892 & 0.000000000 \\ \text { C } & -0.382253283 & -0.617746717 & 0.000000000 \\ \text { C } & 0.382253283 & 0.617746717 & -0.000000000\end{array}$


Cell Parameters:

Note: Cell parameters are in Angstroms
0.000000000
6.113628507
$-6.378705108$
$-0.000000000$
$-6.113628507$
$-6.378705108$
$-29.975832720$
$-0.000000000$
0.000000000 
